\title{
Extended cold snare polypectomy for small colorectal polyps increases the $R 0$ resection rate
}

\section{(ㄷ)(1) $\odot$}

\author{
Authors \\ Shinozaki ${ }^{3,4}$ \\ Institutions \\ 1 Department of Gastroenterology, Department of \\ Medicine, Utsunomiya Memorial Hospital, Tochigi, Japan \\ 2 Department of Surgery, Jichi Medical University, Tochigi, \\ Japan \\ 3 Shinozaki Medical Clinic, Tochigi, Japan \\ 4 Division of Gastroenterology, Department of Medicine, \\ Jichi Medical University, Tochigi, Japan
}

Yasuhiro Abe ${ }^{1}$, Haruaki Nabeta ${ }^{1}$, Ryota Koyanagi ${ }^{1}$, Taro Nakamichi ${ }^{1}$, Hayato Hirashima ${ }^{1}$, Alan Kawarai Lefor ${ }^{2}$, Satoshi

submitted 17.7.2017

accepted after revision 29.11.2017

\author{
Bibliography \\ DOI https://doi.org/10.1055/s-0043-125312 | \\ Endoscopy International Open 2018; 06: E254-E258 \\ (C) Georg Thieme Verlag KG Stuttgart · New York \\ ISSN 2364-3722
}

Corresponding author

Satoshi Shinozaki, MD, PhD, Shinozaki Medical Clinic, 6-1-13 Kiyoharadai, Utsunomiya, Tochigi, 321-3223, Japan Fax: +81-28-667-7305

shinozaki-s@aqua.ocn.ne.jp

\section{ABSTRACT}

Background and study aims Despite widespread use of cold snare polypectomy (CSP), the R0 resection rate is not well documented. We perform extended CSP, resecting polyps with a $>1 \mathrm{~mm}$ circumferential margin. The aim of this study is to compare the $\mathrm{R} 0$ resection rate of extended CSP with conventional CSP and to assess safety.

Patients and methods From April 2014 to September 2016, 712 non-pedunculated colorectal polyps, $<10 \mathrm{~mm}$ in size, resected using CSP from 316 patients were retrospectively analyzed.

Results We divided lesions into conventional CSP $(n=263)$ and extended CSP groups ( $n=449)$. The baseline characteristics of these two groups were not significantly different in univariate or multivariate analyses. Sessile polyps comprised $94 \%$ (668/712), and the remaining were flat-elevated polyps. Mean size of polyps ( \pm standard deviation) was $4.2 \pm 1.5 \mathrm{~mm}$. The most frequent pathology was low grade adenoma (97\%, 689/712). The R0 resection rate was significantly higher in the extended CSP group (439/449 [98\%]) than in the conventional CSP group (222/263 [84\%], $P<$ 0.001 ). There was no delayed bleeding or perforation in either group (conventional CSP group, 0/263, 95\% confidence interval: $0.0-1.4 \%$ and extended CSP group, 0/449, $95 \%$ confidence interval: $0.0-0.8 \%)$.

Conclusions Extended CSP results in a higher $\mathrm{R} 0$ resection rate compared with conventional CSP. Extended CSP did not result in a higher rate of delayed bleeding or perforation. Extended CSP is a safe and promising procedure for endoscopic resection of non-pedunculated colorectal polyps $<10 \mathrm{~mm}$ in size

\section{Introduction}

Endoscopic resection of colorectal polyps decreases the incidence of colorectal cancer and mortality [1]. Although snare polypectomy with electrocautery, referred to as hot snare polypectomy (HSP), has been widely used, an approximately $1 \%$ to $2 \%$ delayed bleeding rate is inevitable, even when performed by well-trained endoscopists [2-4]. Cold snare polypectomy (CSP) has been used for the endoscopic resection of small colorectal polyps in western and eastern countries. CSP does not use electrocautery, with a diminished risk of delayed bleeding and perforation [5]. A low incidence of delayed bleeding and safety of
CSP has been reported in Japan [6, 7]. However, a low rate of R0 resection has also been reported [8].

During the introduction of CSP, we performed CSP for colorectal polyps without involving a wide margin of surrounding normal mucosa to minimize the risk of delayed bleeding and perforation. However, we became concerned about the low R0 resection rate during this introductory period. Since 2015, we perform extended CSP with a more than $1 \mathrm{~mm}$ resection margin for the purpose of increasing the $\mathrm{R} 0$ resection rate. The aim of this study is to compare the R0 resection rate of extended CSP with conventional CSP and to assess the safety of extended CSP. 

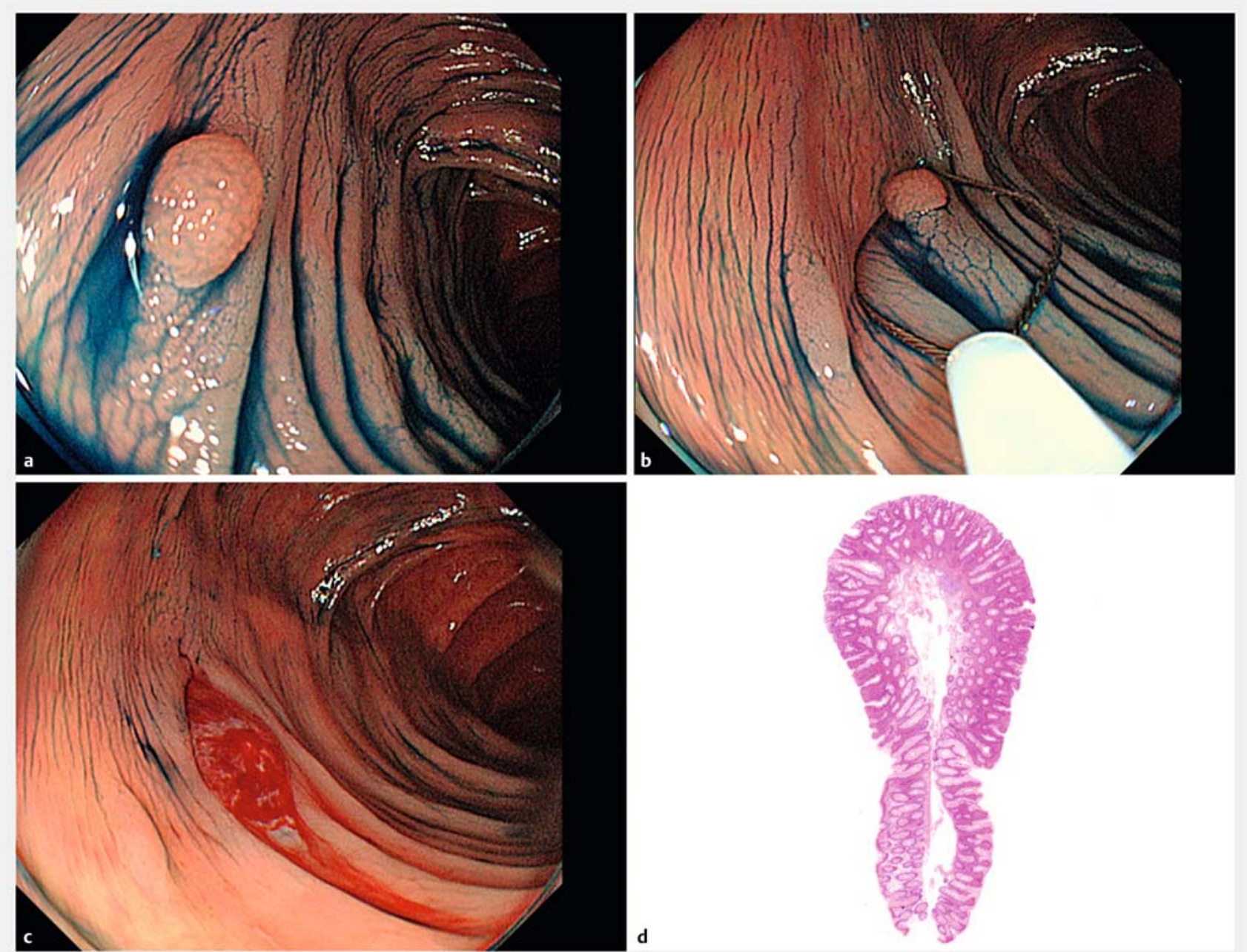

- Fig. 1 Procedure of extended cold snare polypectomy (CSP). a 4-mm sessile polyp in the transverse colon. b Polypectomy snare targets the polyp with $>1 \mathrm{~mm}$ surrounding normal mucosa. $\mathbf{c}$ The mucosal defect after extended CSP; and $\mathbf{d}$ Pathological specimen shows an adequate negative margin.

\section{Patients and methods}

\section{Study population}

A retrospective review of 1304 consecutive polyps in 542 patients who underwent CSP for the treatment of colorectal polyps at the Utsunomiya Memorial Hospital (Utsunomiya, Japan) from April 2014 to September 2016 was performed. CSP was not performed for polyps with suspected carcinoma based on endoscopic findings. Data were collected from medical records, photographs and video recordings. Pedunculated polyps, $\geq 10 \mathrm{~mm}$ in size, undetermined resection method, hyperplastic and inflammatory polyp were excluded from this analysis. This retrospective study was approved by the Institutional Review Board prior to commencement.

\section{CSP procedure}

We divided the polyps into a conventional CSP group and an extended CSP group. The definition of extended CSP was a polyp resected with a more than $1 \mathrm{~mm}$ circumferential margin ( Fig. 1). We defined others as conventional CSP. An R0 resection was defined as an en bloc resection with pathologically negative resection margins [9]. Because of the low R0 resection rate using conventional CSP during the introductory period of CSP, we performed extended CSP since May 2015 to increase the $R 0$ resection rate. Since that time, extended CSP was always attempted, however conventional CSP was occasionally performed because of technical problems due to location and peristalsis. Consequently, we mainly performed conventional CSP in the early phase of the study period, and performed extended CSP during the later period. The PCF-Q260AZI or CF-Q260AI (Olympus, Tokyo, Japan) was used. The polypectomy snare for CSP was an $11 \mathrm{~mm}$, Extra Small Oval-Flexible, CAPTIVATOR ${ }^{\text {TM }}$ II (Boston Scientific, Natick, MA, USA) or $10 \mathrm{~mm}$, Oval, SnareMaster $^{\mathrm{TM}}$ (Olympus, Tokyo, Japan). The resected polyp was suc- 
- Table 1 Study patient characteristics.

\begin{tabular}{|l|c|c|c|}
\hline & $\begin{array}{l}\text { Conventional } \\
\text { CSP group }\end{array}$ & $\begin{array}{l}\text { Extended } \\
\text { CSP group }\end{array}$ & P value \\
\hline Patients, n & 119 & 197 & \\
\hline Males, n & $99(83 \%)$ & $149(76 \%)$ & 0.113 \\
\hline Age (years), mean \pm SD & $60.7 \pm 10.7$ & $61.4 \pm 10.4$ & 0.639 \\
\hline SD: standard deviation, CSP: cold snare polypectomy & \\
\hline
\end{tabular}

tioned and retrieved after CSP. Clip application was not performed. The retrieved polyp was preserved in formalin and assessed by routine histopathologic studies. The definition of delayed bleeding was overt bleeding within two weeks after the CSP.

\section{Statistical analysis}

Categorical data were compared by using the chi-squared test. Data with a normal distribution were compared with an independent t-test. To estimate the $95 \%$ confidence intervals $(\mathrm{Cl})$ around the estimated proportions of patients with adverse events, we used the Clopper-Pearson exact method. Differences were considered to be statistically significant when $P<$ 0.05 . The above statistical analyses were performed using BellCurve for Excel 2015 software (Social Survey Research Information Co., Ltd. Tokyo, Japan). To evaluate potential confounding factors before resection, we used logistic regression analysis for multivariate analysis, performed using Statflex ver. 6.0 software (Artech Co. Ltd. Osaka, Japan).

\section{Results}

\section{Study population}

Based on the exclusion criteria, 592 polyps in 226 patients were excluded as follows: pedunculated polyp $(n=327), \geq 10 \mathrm{~mm}(\mathrm{n}=$ $38)$, undetermined resection method $(n=51)$, hyperplastic $(n=$ 168 ) and inflammatory polyp $(n=8)$. Consequently, data for 712 sessile colorectal polyps in 316 patients were included in this final analysis. The characteristics of these 316 patients are shown in - Table 1. Seventy-eight of the patients were male and the mean age was 61 . We divided them into conventional CSP $(n=119)$ and extended CSP groups $(n=197)$. Male gender was slightly more common in the conventional CSP group than in the extended CSP group $(P=0.113)$, and mean age was similar in both groups $(P=0.592)$.

\section{R0 resection rate}

The characteristics of 712 polyps are shown in $>$ Table 2 . We divided them into conventional CSP $(n=263)$ and extended CSP groups $(n=449)$. The baseline characteristics before treatment of these two groups were not significantly different in univariate and multivariate analyses ( $>$ Table 2 and $\triangleright$ Table 3 ). Sessile polyps comprised $94 \%$ (668/712), and the remaining were flat-elevated polyps. The mean size of the polyps ( \pm standard deviation) was $4.2 \pm 1.5 \mathrm{~mm}$. The most frequent pathology
- Table 2 Characteristics of 712 colorectal polyps resected by cold snare polypectomy (CSP).

\begin{tabular}{|c|c|c|c|}
\hline & $\begin{array}{l}\text { Conventional } \\
\text { CSP group }\end{array}$ & $\begin{array}{l}\text { Extended } \\
\text { CSP group }\end{array}$ & $\begin{array}{l}P \text { val- } \\
\text { ue }\end{array}$ \\
\hline Resected polyps, n & 263 & 449 & \\
\hline \multicolumn{4}{|l|}{ Location, n } \\
\hline - Right colon & $134(51 \%)$ & $244(54 \%)$ & 0.381 \\
\hline - Left colon & $109(41 \%)$ & $167(37 \%)$ & 0.261 \\
\hline - Rectum & $20(8 \%)$ & $38(9 \%)$ & 0.686 \\
\hline \multicolumn{4}{|l|}{ Shape, n } \\
\hline - Sessile & $242(92 \%)$ & $426(95 \%)$ & 0.126 \\
\hline - Flat-elevated & $21(8 \%)$ & $23(5 \%)$ & \\
\hline \multicolumn{4}{|l|}{ Size (mm), n } \\
\hline - $1-4 \mathrm{~mm}$ & 140 & 272 & 0.055 \\
\hline . $5-9 \mathrm{~mm}$ & 123 & 177 & \\
\hline \multicolumn{4}{|l|}{ Pathology, n } \\
\hline - Serrated polyp & $10(4 \%)$ & $7(1 \%)$ & 0.058 \\
\hline - Low-grade adenoma & $253(96 \%)$ & $436(97 \%)$ & 0.508 \\
\hline - High-grade adenoma & $0(0 \%)$ & $3(1 \%)$ & 0.184 \\
\hline - Adenocarcinoma & $0(0 \%)$ & $3(1 \%)$ & 0.184 \\
\hline \multicolumn{4}{|l|}{ Adverse events, $\mathrm{n}$} \\
\hline - Delayed bleeding & $0(0 \%)$ & $0(0 \%)$ & \\
\hline - Perforation & $0(0 \%)$ & $0(0 \%)$ & \\
\hline SD, standard deviation & & & \\
\hline
\end{tabular}

- Table 3 Evaluation of pretreatment factors for selection of extended cold snare polypectomy (multivariate analysis).

\begin{tabular}{|l|l|l|l|}
\hline Factor & $\begin{array}{l}\text { Odds } \\
\text { ratio }\end{array}$ & $\begin{array}{l}\text { 95\% confidence } \\
\text { interval }\end{array}$ & $\begin{array}{l}\boldsymbol{P} \text { val- } \\
\text { ue }\end{array}$ \\
\hline Location: Right colon & 0.970 & $0.541-1.738$ & 0.919 \\
\hline Location: Left colon & 0.826 & $0.455-1.497$ & 0.529 \\
\hline Shape: Sessile polyp & 1.438 & $0.765-2.702$ & 0.258 \\
\hline Size: $5-9 \mathrm{~mm}$ & 0.780 & $0.568-1.071$ & 0.125 \\
\hline
\end{tabular}

was low grade adenoma $(97 \%, 689 / 712)$. The $\mathrm{R} 0$ resection rate was significantly higher in the extended CSP group (439/449 [98\%]) than in the conventional CSP group (222/263 [84\%], $P<0.001$ ) (> Fig.2).

\section{Adverse events}

The overall rate of adverse events was $0 \%$ per procedure $(0 /$ $712,95 \% \mathrm{Cl}: 0.0-0.52 \%)$ and $0 \%$ per patient $(0 / 316,95 \% \mathrm{Cl}$ : $0.0-1.16 \%)$. The rates of delayed bleeding and perforation in the conventional CSP group were $0 \%$ per procedure $(0 / 263$, $95 \% \mathrm{Cl}: 0.0-1.39 \%)$ and $0 \%$ per patient $(0 / 119,95 \% \mathrm{Cl}: 0.0-$ 


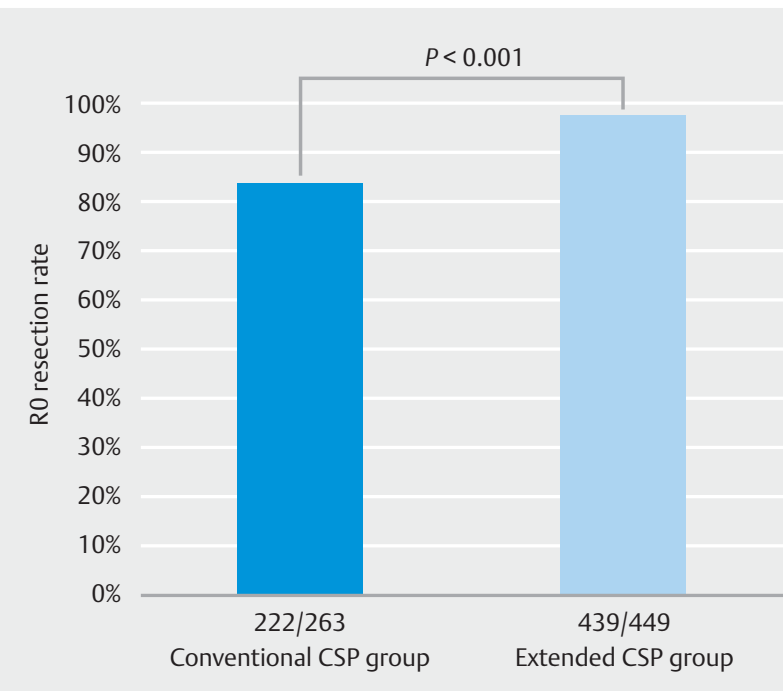

- Fig. 2 Comparison of R0 resection rate between the conventional cold snare polypectomy (CSP) group and the extended CSP group.

$3.05 \%)$. In the extended CSP group, delayed bleeding and perforation were also $0 \%$ per procedure $(0 / 449,95 \% \mathrm{Cl}: 0.0-$ $0.82 \%)$ and $0 \%$ per patient (0/197, $95 \% \mathrm{Cl}: 0.0-1.86 \%)$.

\section{Discussion}

This study shows that extended CSP has a significantly higher rate of R0 resection compared to conventional CSP for nonpedunculated colorectal polyps $<10 \mathrm{~mm}$. The safety of extended CSP is also demonstrated. CSP, which started in Western countries, is becoming more popular in Eastern countries as a simple and safe approach for resecting small colorectal polyps [5]. Since the malignant potential of small colorectal polyps is low, an almost zero rate of adverse events including delayed bleeding and perforation is important. CSP is suitable for the treatment of small colorectal polyps, because it has a shorter procedure time and lower rate of delayed bleeding than HSP or endoscopic mucosal resection $[7,10]$. The present study, which includes a large number of small non-pedunculated colorectal polyps, demonstrates that extended CSP is a promising method to increase the $\mathrm{R} 0$ resection rate.

A low $\mathrm{R} 0$ resection rate (59\%) of small polyps resected by CSP has been reported [8]. We suggest two explanations for a low $\mathrm{R} 0$ resection rate. First, in preparing the specimen for pathological examination, determination of the exact margin for specimens resected by CSP is difficult because of a macroscopically unclear resection margin, since, unlike HSP, there is no cauterized area after CSP. Second, CSP makes a pathological diagnosis difficult microscopically because the resection margin of small colorectal polyps is not cauterized. Taken together, a resected polyp with adequate surrounding normal colonic mucosa is helpful to recognize the resection margin and to improve the quality of the pathological diagnosis.

Although intra-procedural bleeding is generally more common in CSP compared with HSP, the colonoscopist is rarely con- cerned with intra-procedural bleeding. Unlike delayed bleeding, persistent intra-procedural bleeding is easily controlled by applying a clip. CSP rarely leads to delayed ulceration after resection because of the lack of thermal injury. Anatomically, the submucosa has larger arteries and veins than the lamina propria. Delayed bleeding after HSP is usually caused by vascular damage in the submucosal layer due to use of the electrocautery. Generally, the rate of delayed bleeding after HSP is reportedly $1-2 \%[4,11]$, and the benefit of prophylactic clip application after HSP remains controversial $[12,13]$. When performing HSP, an extended mucosal resection without injection may involve the deep submucosa or muscularis, resulting in perforation. As reported by Horiuchi et al [6], CSP is less associated with injuries to the submucosa than HSP. Tuttici et al reported the characteristics of protrusions within the cold snare defect including submucosa and muscularis mucosa without an adenomatous component or vascular structure [14]. We believe that extended CSP may not damage the vessels in the submucosal layer. Therefore, extended CSP does not increase the rate of delayed bleeding and perforation. The $95 \% \mathrm{Cl}$ for the rate of delayed bleeding in the extended CSP group in the present study was less than $1 \%$.

For the treatment of colorectal tumors, an en bloc R0 resection is important to determine the completeness of resection and to decrease the rate of local recurrence [5]. En bloc resection is defined as a tumor removed in one piece, and $\mathrm{R} 0$ resection is defined as an en bloc resection with negative pathologic margins. Although endoscopic resection of small colorectal polyps is usually straightforward, local recurrence after polypectomy necessitates additional resection performed by CSP, HSP, endoscopic mucosal resection or endoscopic submucosal dissection. Unlike the first endoscopic treatment, when treating a recurrent lesion one frequently encounters severe submucosal fibrosis due to the first resection, making the resection difficult. To decrease the need for additional resection, the extended CSP is a viable option. Further, we recognize that CSP involving surrounding normal mucosa has become a standard strategy [15]. The technique referred to here as an "extended CSP" may represent the standard technique advocated by experts used in the clinical practice of CSP. However, to date, there is no significant evidence reporting the superiority of this extended CSP strategy over conventional CSP. Although the pathological significance of extended CSP compared with conventional CSP should ideally be shown by a randomizedcontrolled trial, the superiority of extended CSP is obvious and such a clinical trial may be difficult to conduct.

We recognize some acknowledged limitations of this study. First, the study is limited by its retrospective design and several potential biases. Both techniques were compared indirectly in two different periods and the impact of the learning curve of the endoscopists and pathologists cannot be excluded. The pathologists who assessed completeness of resection could not be blinded to the procedure performed. However, an extended CSP is reasonable to improve the $\mathrm{R} 0$ resection rate, and no adverse events occurred in this study including a large number of CSP procedures. Second, there is no evaluation of local recurrence after CSP in this study. The rate of local recurrence after 
CSP as a long-term outcome remains unclear [7, 15]. Third, this study did not include pedunculated polyps but only non-pedunculated polyps $<10 \mathrm{~mm}$ in size. Therefore, this result should not be extrapolated to CSP for pedunculated colorectal polyps. Generally, pedunculated polyp have a large vessel with greater potential for delayed bleeding [16].

\section{Conclusion}

In conclusion, this study shows that extended CSP results in a higher R0 resection rate for non-pedunculated colorectal polyps $<10 \mathrm{~mm}$ compared with conventional CSP. Extended CSP did not result in a higher rate of delayed bleeding or perforation. To our knowledge, this is the first report of the safety and efficacy of extended CSP in compared with conventional CSP. Extended CSP is a safe and promising procedure for endoscopic resection of non-pedunculated colorectal polyps $<10$ $\mathrm{mm}$ in size.

Competing interests

None

\section{References}

[1] Zauber AG, Winawer S], O'Brien M] et al. Colonoscopic polypectomy and long-term prevention of colorectal-cancer deaths. N Engl J Med 2012; 366: 687-696

[2] Heldwein W, Dollhopf M, Rosch T et al. The Munich Polypectomy Study (MUPS): prospective analysis of complications and risk factors in 4000 colonic snare polypectomies. Endoscopy 2005; 37: 1116 1122

[3] Rex DK, Bond JH, Winawer S et al. Quality in the technical performance of colonoscopy and the continuous quality improvement process for colonoscopy: recommendations of the U.S. Multi-Society Task Force on Colorectal Cancer. Am J Gastroenterol 2002; 97: 1296 1308
[4] Tanaka S, Kashida H, Saito Y et al. JGES guidelines for colorectal endoscopic submucosal dissection/endoscopic mucosal resection. Dig Endosc 2015; 27: 417-434

[5] Shinozaki S, Hayashi Y, Lefor AK et al. What is the best therapeutic strategy for colonoscopy of colorectal neoplasia? Future perspectives from the East Dig Endosc 2016; 28: 289-295

[6] Horiuchi A, Nakayama Y, Kajiyama M et al. Removal of small colorectal polyps in anticoagulated patients: a prospective randomized comparison of cold snare and conventional polypectomy. Gastrointest Endosc 2014; 79: 417-423

[7] Ichise Y, Horiuchi A, Nakayama Y et al. Prospective randomized comparison of cold snare polypectomy and conventional polypectomy for small colorectal polyps. Digestion 2011; 84: 78-81

[8] Takeuchi Y, Yamashina T, Matsuura N et al. Feasibility of cold snare polypectomy in Japan: A pilot study. World J Gastrointest Endosc 2015; 7: $1250-1256$

[9] Sakamoto H, Hayashi Y, Miura Y et al. Pocket-creation method facilitates endoscopic submucosal dissection of colorectal laterally spreading tumors, non-granular type. Endosc Int Open 2017; 5: E123-E129

[10] Uraoka T, Ramberan H, Matsuda T et al. Cold polypectomy techniques for diminutive polyps in the colorectum. Dig Endosc 2014; 26: 98 103

[11] Oka S, Tanaka S, Kanao H et al. Current status in the occurrence of postoperative bleeding, perforation and residual/local recurrence during colonoscopic treatment in Japan. Dig Endosc 2010; 22: 376 380

[12] Shioji K, Suzuki Y, Kobayashi M et al. Prophylactic clip application does not decrease delayed bleeding after colonoscopic polypectomy. Gastrointest Endosc 2003; 57: 691-694

[13] Friedland S, Soetikno R. Colonoscopy with polypectomy in anticoagulated patients. Gastrointest Endosc 2006; 64: 98 - 100

[14] Tutticci N, Burgess NG, Pellise M et al. Characterization and significance of protrusions in the mucosal defect after cold snare polypectomy. Gastrointest Endosc 2015; 82: 523-528

[15] Hewett DG, Rex DK. Colonoscopy and diminutive polyps: hot or cold biopsy or snare? Do I send to pathology? Clin Gastroenterol Hepatol 2011; 9: $102-105$

[16] Kwon MJ, Kim YS, Bae SI et al. Risk factors for delayed post-polypectomy bleeding. Intest Res 2015; 13: 160-165 